\title{
A Survey on IOT based Real Time, Smart Adaptive Street Lighting System with Pollution Monitoring for Smart Cities
}

\author{
Supreetha D \\ Department of CSE \\ Global Academy of Technology \\ Bangalore-560098 \\ supreethad@gmail.com
}

\author{
Vinay Sagar K S \\ Department of CSE \\ Global Academy of Technology \\ Bangalore-560098 \\ ksvinay043@gmail.com
}

\author{
Sushmitha S \\ Department of CSE \\ Global Academy of Technology \\ Bangalore-560098 \\ sushmitha.s15@gmail.com
}

\begin{abstract}
The Internet of Things (IoT) is a new interconnection of technology. It is being heralded as the next industrial revolution. It's also praised for its ability to reduce waste and energy, by allowing us to better control our use of resources based on our actual needs. Currently, energy consumed by the street lights in metropolitan cities is extremely high and hence are less efficient. One of the major problems with the existing street lighting system is that all the street lights are controlled manually and therefore require huge manpower. Even if a street light stops working there is no way by which the authorities can know about the failure. Another growing concern in cities is the pollution.

The purpose of this paper is to propose a solution which can be adopted in every city where all the street lights are connected to the central server via internet which can be monitored remotely and thus making it an energy efficient system. These street lights are adaptive meaning that they can change the intensity of light depending on the vehicular movement. They also send information about the pollution level in that locality. Thus IoT promises huge improvements in street lighting system.
\end{abstract}

Keywords- Energy efficient system, NodeMCU, Android phone application, Pollution monitoring, Motion detection sensor.

$* * * * *$

\section{INTRODUCTION}

The Internet of Things [Fig.1] is the network of physical devices, vehicles, home appliances and other embedded with electronics, software, sensors, actuators, and connectivity which enable these objects to connect and exchange data. Each thing is uniquely identifiable through its embedded computing system but is able to inter-operate within the existing Internet infrastructure. The Internet of Things is the expansion of the current Internet services so as to accommodate each and every object which exists in this world or likely to exist in the coming future.

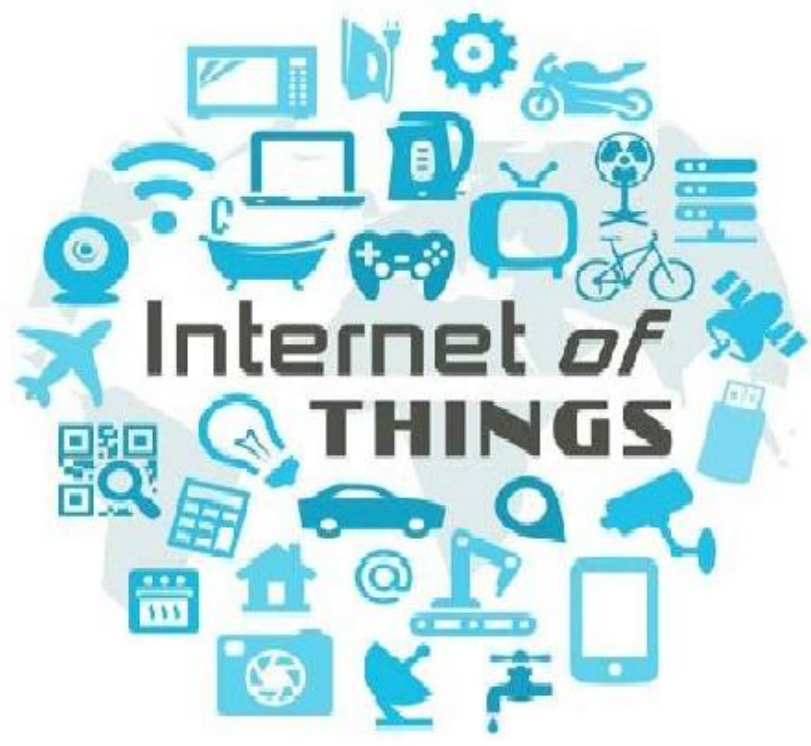

Fig.1 Internet of Things
The transformation from a classic street lighting system to a smart street lighting solution is primarily enabled by the ability of devices to connect to and communicate with each other. It means that the various components of a normal street lighting system can be interlinked via a communication network. Such connected systems overcome the limitations of manual systems and allows one to remotely switch ON or switch OFF street lights, control parameters like intensity of individual street light, detect and report faults from remote locations, identify the presence of pedestrians or vehicles to switch ON lights, measure the power consumed by streetlight, monitoring additional parameters like air pollution, temperature and humidity by using various sensors [1]. All the sensors are placed on the lamp pole and the efficiency of these sensors on the lamp are affected by sensing area, height of lamp pole, angle at which the sensor is kept etc. Pedestrians and vehicles on road are sensed and information about their movement is collected in a sensor array [5]. This information is transferred pointby-point using microcontroller transmitters and receivers which are then sent to a control terminal that is used to check the state of the street lamps.

A Web and Android application is used to individually monitor and control the street lights. All the information about the temperature, humidity, air pollution levels and status of streetlight is present in the application [3]. 
Every Street light will have a unique number and address embedded into it. In case of its malfunctioning, its address will be sent to the authorities so that they can repair it [4]. The system allows energy savings with increased performance. According to "100 Smart Cities Mission" which was launched by our Prime Minister Narendra Modi on 25 June 2016 a total of 98,000 crore (US $\$ 14$ billion) has been approved by the Indian Cabinet for the development of 100 smart cities and the rejuvenation of 500 others. It is a five-year program in which all of the Indian states and Union territories are participating by nominating at least one city for the Smart Cities challenge. Financial aid will be given by the central and state governments between 2017-2022 to the cities.

\section{IOT ARCHITECTURE}

IoT architecture can be explained as a network of things which consists of hardware, software, network connectivity, and sensors. Hence, the architecture of IoT comprises of four major components: sensing, network, data processing, and application layers [Fig.2]. A detailed description of these layers is given below.

\section{A. Sensing layer}

The main purpose of the sensing layer is to identify any phenomena in the device's peripheral and obtain data from the real world. This layer consists of several sensors. Using multiple sensors for applications is one of the primary features of IoT devices. Sensors in IoT devices are usually integrated through sensor hubs. A sensor hub is a common connection point for multiple sensors that accumulate and forward sensor data to the processing unit of a device. A sensor hub uses several transport mechanisms (Inter-Integrated Circuit (I2C) or Serial Peripheral Interface (SPI)) for data flow between sensors and applications. These transport mechanisms depend on IoT devices and create a communication channel between the sensors and the applications to collect sensor data. Sensors in IoT devices can be classified in three broad categories as described below.

1. Motion Sensors- Motion sensors measure the change in motion as well as the orientation of the devices. There are two types of motions one can observe in a device: linear and angular motions. The linear motion refers to the linear displacement of an IoT device while the angular motion refers to the rotational displacement of the device.

2. Environmental Sensors- Sensors such as Light sensor, Pressure sensor, etc. are embedded in IoT devices to sense the change in environmental parameters in the device's peripheral. The primary purpose of using environmental sensors in IoT devices is to help the devices to take autonomous decisions according to the changes of a device's peripheral. For instance, environment sensors are used in many applications to improve user experience (e.g., home automation systems, smart locks, smart lights, etc.).

3. Position sensors- Position sensors of IoT devices deal with the physical position and location of the device. Most common position sensors used in IoT devices are magnetic sensors and Global Positioning System (GPS) sensors. Magnetic sensors are usually used as digital compass and help to fix orientation of device display. On the other hand, GPS is used for navigation purposes in IoT devices.

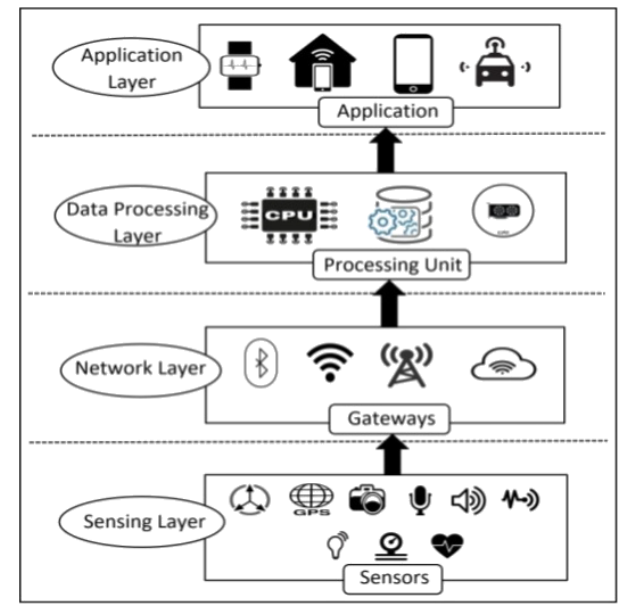

Fig.2 IoT Architecture Layers and Components

\section{B. Network Layer}

The network layer acts as a communication channel to transfer data, collected in the sensing layer, to other connected devices. In IoT devices, the network layer is implemented by using diverse communication technologies (e.g., Wi-Fi, Blue-tooth, Zigbee, Z-Wave, LoRa, cellular network, etc.) to allow data flow between other devices within the same network.

\section{Data Processing Layer}

The data processing layer consists of the main data processing unit of IoT devices. The data processing layer takes data collected in the sensing layer and analyses the data to take decisions based on the result. In some IoT devices (e.g., smart watch, smart home hub, etc.), the data processing layer also saves the result of the previous analysis to improve the user experience. This layer may share the result of data processing with other connected devices via the network layer.

\section{Application Layer}

The application layer implements and presents the results of the data processing layer to accomplish disparate applications of IoT devices. The application layer is a user-centric layer which executes various tasks for the 
users. There exist diverse IoT applications, which include smart transportation, smart home, personal care, healthcare, etc.

\section{KEY CHARACTERISTICS FOR IOT}

The fundamental characteristics of the IoT are as follows-

1. Interconnectivity- With regard to the IoT, anything can be interconnected with the global information and communication infrastructure.

2. Things-related services- The IoT is capable of providing thing-related services within the constraints of things, such asprivacy protection and semantic consistency between physical things and their associated virtual things. In order to providething-related services within the constraints of things, both the technologies in physical world and information world willchange.

3. Heterogeneity- The devices in the IoT are heterogeneous as based on different hardware platforms and networks. They can interact with other devices or service platforms through different networks.

4. Dynamic changes- The state of devices change dynamically,e.g., sleeping and waking up, connected and/or disconnectedas well as the context of devices including location and speed.Moreover, the number of devices can change dynamically.

5. Enormous scale- The number of devices that need to bemanaged and that communicate with each other will be at leastan order of magnitude larger than the devices connected to thecurrent Internet. Even more critical will be the management ofthe data generated and their interpretation for applicationpurposes.

6. Safety- As we gain benefits from the IoT, we must notforget about safety. As both the creators and recipients of theIoT, we must design for safety. This includes the safety of our personal data and the safety of our physical well-being.Securing the endpoints, the networks, and the data moving across all of it means creating a security paradigm that will scale.

7. Connectivity- Connectivity enables network accessibility and compatibility. Accessibility is getting on a network while compatibility provides the common ability to consume and produce data.

\section{LIMITATIONS OF EXISTING SYSTEM}

As a matter of fact, there is trending demand for making efficient street lights upgraded with latest technology to meet the growing demands of the people. The current street lighting policy requires all lights to be fully operational during the entire night, due to security reasons and inadequate diming technology. Mainly for urban cities most of the power is wasted in lighting up the streets when there is a minimum traffic flow during late nights. This leads to unnecessary energy use, lowers the lamps life and causes significant light pollution. Considering the above problems of conventional lighting methods, it has become increasingly important to develop a radically new system that is both environmental friendly and cost effective. In our nature, pollution is one of the biggest problems. It occurs because of impurities mix with the air, water and soil. To keep our nature safe, we need to keep track of pollution level of air. As we all know "Prevention is better than cure", we need to be alert before the pollution level cross safe limits. The following are the issues of the existing system.

\section{A. Manual Switching}

This is the classic and omnipresent technique. The light is switched $\mathrm{ON}$ and $\mathrm{OFF}$ by a human attendant. Thus the street lights are controlled manually and therefore require huge manpower.

B. Energy Consumption is very high

Conventional street lighting system use constant illumination lighting which leads to high energy consumption accounting for up to $60 \%$ of a municipal government's total electricity expenditure. Furthermost, forecast show that the energy spending for street lights is likely to increase over the next few years as the demand and price for electricity increases.

C. Maintaince Problem

One of the major problem with the existing system is that even if a street light stops working there is no way by which the authorities can know about the failure. It takes 3-4 days on average for the authorities to identify the non functional street light and repair it.

D. Does not posses smart features

In current scenario, the street lights do not posses smart features like pollution monitoring and adaptive nature. The term adaptive nature means the street lights can change the intensity of light depending on the vehicular movement.

V. IOT FOR STREET LIGHTING SYSTEM

The IoT street lighting system consists of threelayered hierarchical architecture shown in Fig.3. 


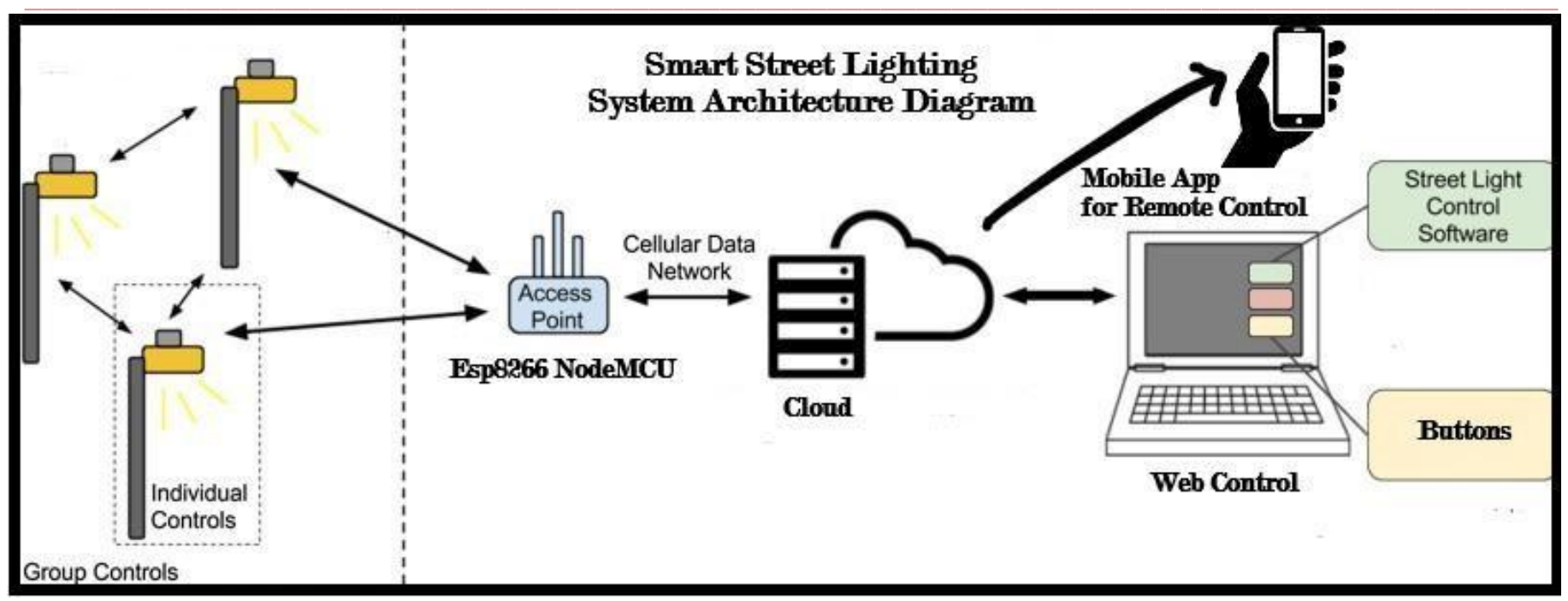

Fig.3 IoT for street lighting system

The lower layer is composed by end nodes installed on street lights to perform sensing and measurements. The second layer of the hierarchy provides connectivity to both remote servers of control systems and the end nodes. The highest level of the hierarchy is the application layer that consists of the remote control system or server, whose task includes optimizing management and efficiency of street lighting systems and performs other applicable strategies.

The four blocks of Street lighting system are sensors and a processor, cloud platform, OLED display and a mobile application. The block diagram of street lighting system is shown in Fig.4.

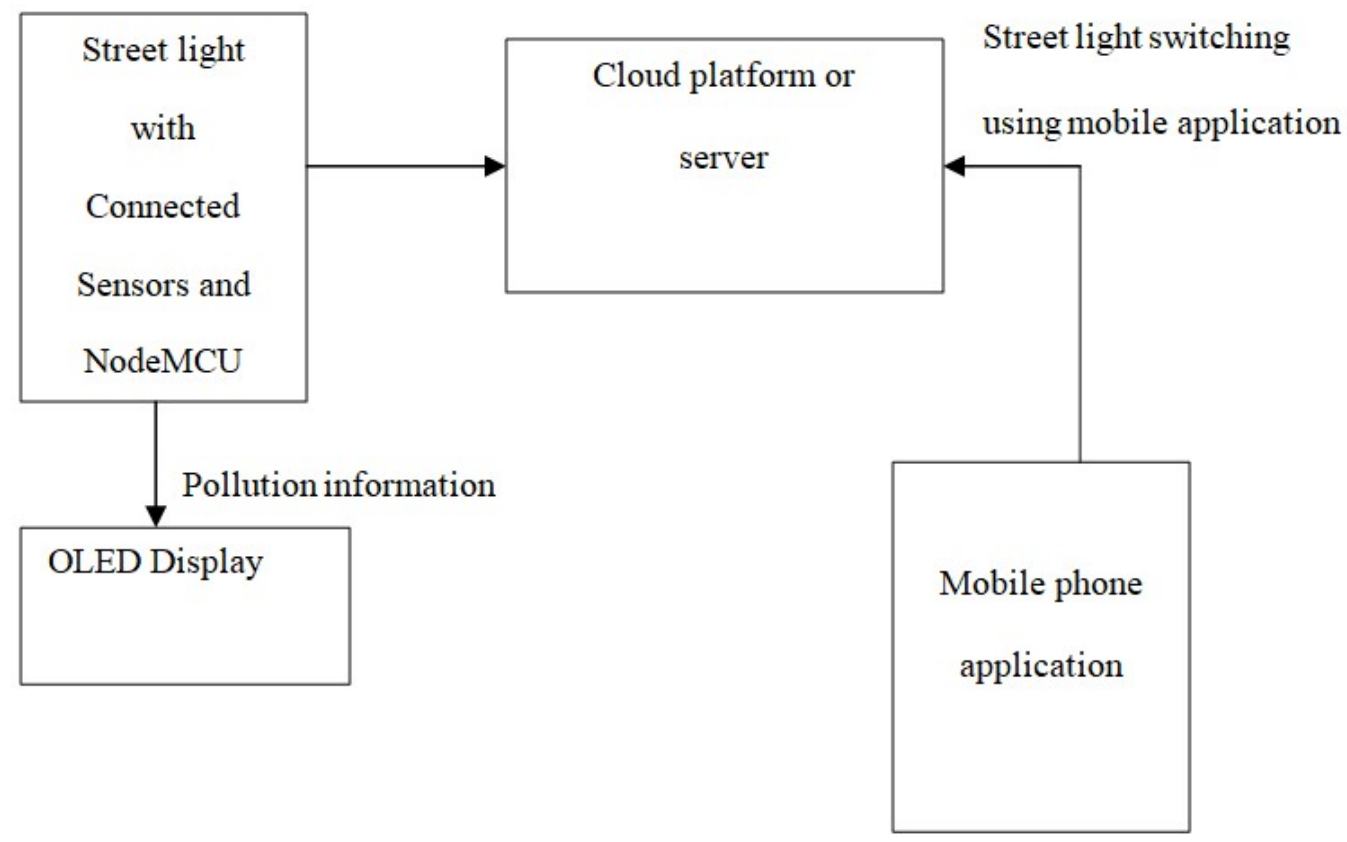

Fig. 4 Block diagram of street lighting system

From the block diagram, we have a street light which is made into a smart street light using sensors such as LDR (light dependent resistor), temperature and humidity sensor, air quality sensor and a motion detection sensor. The temperature and humidity sensor and the air quality sensor values are displayed on the OLED display. While the LDR and the motion detection sensors are used to turn ON and turn OFF the street light.
Based on the intensity of the sunlight, the LDR output value changes and hence we can turn ON the street light during darkness and vice versa. Whereas the motion detection sensor senses the motion of vehicles and can control the intensity of light. All these methods to trigger the switching of street lights make them more energy efficient. 
Another feature implemented in this project is usage of Android phone application to perform the street light switching action via cloud or servers.

\section{TECHNOLOGIES AND DEVICES}

The following technologies and devices used in this work are briefly described.

\section{A. ESP8266 NodeMCU}

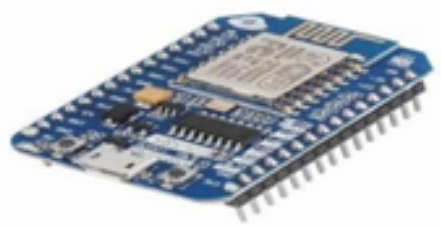

Fig.5 ESP8266 NodeMCU

NodeMCU [Fig.5] is an open source LUA based firmware developed for ESP8266 WIFI chip. It includes firmware which runs on the ESP8266 Wi-Fi SoC from Espressif Systems, and hardware which is based on the ESP12 module. With just a few lines of code you can establish a WiFi connection and define input/output pins according to your needs exactly like arduino, turning your ESP8266 into a web server and a lot more. It is the WiFi equivalent of Ethernet module. With its USBTTL,the NodeMCU Dev board supports directly flashing from USB port. It combines features of WIFI access point and station+ microcontroller. These features make the NodeMCU extremely powerful tool for Wifi networking. It can be used as access point and/or station, host a web server or connect to internet to fetch or upload data.

\section{B. PIR Motion Detection Sensor}

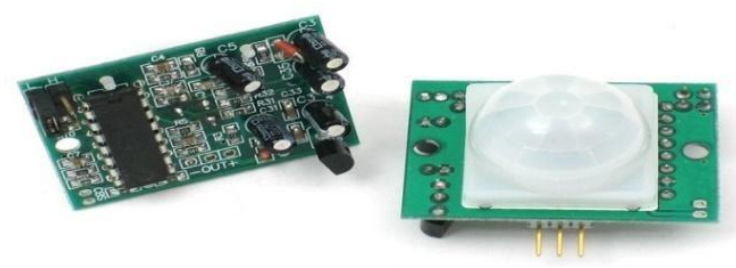

Fig.6 PIR Motion Detection Sensor

PIR sensor [Fig.6] allows you to sense motion, almost always used to detect whether a human has moved in or out of the sensors range. They are small, inexpensive, low-power, easy to use and don't wear out. For that reason they are commonly found in appliances and gadgets used in homes or businesses. They are often referred to as PIR, "Passive Infrared", "Pyroelectric", or "IR motion" sensors.

PIRs are basically made of a pyroelectric sensor, which can detect levels of infrared radiation. Everything emits some low level radiation, and the hotter something is, the more radiation is emitted. The sensor in a motion detector is actually split in two halves. The reason for that is that we are looking to detect motion (change) not average IR levels. The two halves are wired up so that they cancel each other out. If one half sees more or less IR radiation than the other, the output will swing high or low.

\section{Light Dependent Resistor}

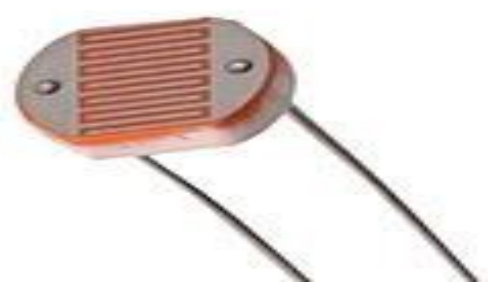

Fig.7 Light Dependent Resistor

Photo resistors [Fig.7] also known as light dependent resistors (LDR), are light sensitive devices most often used to indicate the presence or absence of light, or to measure the light intensity. In the dark, their resistance is very high, sometimes up to $1 \mathrm{M} \Omega$, but when the LDR sensor is exposed to light, the resistance drops dramatically, even down to a few ohms, depending on the light intensity. LDRs have a sensitivity that varies with the wavelength of the light applied and are nonlinear devices. They are used in many applications but are sometimes made obsolete by other devices such as photodiodes and phototransistors. Some countries have banned LDRs made of lead or cadmium over environmental safety concerns.

\section{DHT11 Sensor}

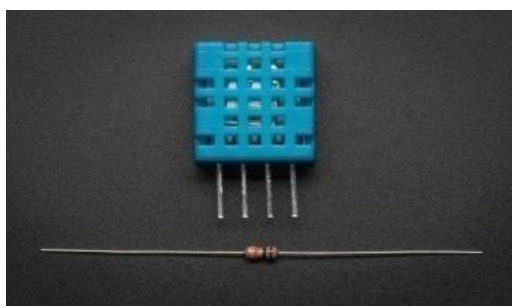

Fig.8 DHT11 Sensor

The DHT11 [Fig.8] is a basic, ultra low-cost digital temperature and humidity sensor. It uses a capacitive humidity sensor and a thermostat to measure the surrounding air, and spits out a digital signal on the data pin (no analog input pins needed). 
E. OLED Display

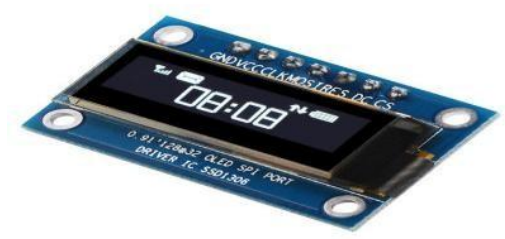

Fig. 9 OLED Display

An organic light-emitting diode [Fig.9] is a lightemitting diode (LED) in which the emissive electroluminescent layer is a film of organic compound that emits light in response to an electric current. This layer of organic layers is situated between two electrodes; typically, at least one of these electrodes is transparent. OLEDs are used to create digital displays in devices such as television screens, computer monitors, portable systems such as smart phones, handheld game consoles and PDAs. A major area of research is the development of white OLED devices for use in solidstate lighting applications.

There are two main families of OLED: those based on small molecules and those employing polymers. An OLED display can be driven with a passive-matrix (PMOLED) or active- matrix (AMOLED) control scheme. In the PMOLED scheme, each row (and line) in the display is controlled sequentially, one by one, whereas AMOLED control uses a thin-film transistor backplane to directly access and switch each individual pixel on or off, allowing for higher resolution and larger display sizes.

\section{CONCLUSION}

This paper "IOT based real time, smart adaptive street lighting system with pollution monitoring for smart cities" is a cost effective, practical, eco-friendly and the safest way to save energy.

In this paper, an overview of the available IoT solutions for street lighting system is provided. It clearly tackles the two problems that world is facing today, high energy consumption by street lighting systems and increase in pollution level. These

suggested techniques remain wide open for the future investigation.

\section{REFERENCES}

[1] Sayali Arkade,Akshada Mohite, Shraddha Joshi, "IOT Based Street Lights for Smart City", International Journal for Research in Applied Science and Engineering Technology(IJRASET), Vol. 4 ,Issue XII, December 2016.

[2] Shagun Malhotra,Vivek Kumar, "Smart Street Lighting System: An Energy Efficient Approach",International
Journal of Science and Research,volume 5 ,Issue 2,February 2016.

[3] Fabio Leccese, Marco Cagnetti and Daniele Trinca, "A Smart City Application: A Fully Controlled Street Lighting Isle Based on Raspberry-Pi Card, a ZigBee Sensor Network and WiMAX”, 18 December 2014.

[4] Reinhard Mullner,Andreas Riener, "An energy efficient pedestrian aware Smart Street Lighting System", International journal of Pervasive Computing and Communications,vol.7,no.2, February 2011.

[5] Samir A.Elsagheer Mohamed, "Smart Street Lightning Control and Monitoring System for Electrical Power Saving by Using VANET',International Journal communications,Network ans System Science(1JCNS), August 2013.

[6] N. Bari, G. Mani, and S. Berkovich, "Internet of things as a method-ological concept," in Fourth International Conference on Computing for Geospatial Research and Application (COM. Geo), 2013. IEEE, pp. 48-55.

[7] N. D. Lane, E. Miluzzo, H. Lu, D. Peebles, T. Choudhury, and A. T. Campbell, "A survey of mobile phone sensing," IEEE Communications magazine, vol. 48, no. 9, 2010. 